\title{
Effect of Error in Measurement of Elastic Constants on the Solutions of Problems in Classical Elasticity
}

\author{
J. H. Bramble* and L. E. Payne*
}

(April 8, 1963)

\begin{abstract}
It is well known that a small error in the measurement of the elastic constants will result, for all physically interesting boundary value problems, in small errors in the computed values of the stresses and displacements. In this paper actual bounds are given for the error in both the first and second boundary value problems. In addition it is shown that as Poisson's ratio tends to $1 / 2$ the results for compressible theory tend to those for the analogous problems in the classical incompressible theory.
\end{abstract}

\section{Introduction}

In the boundary value problems of classical elasticity the elastic constants of the material in question are assumed known. The solution of the problem is then expressed in terms of the "known" elastic constants. However, the values of these elastic constants are actually determined empirically and are therefore not known exactly. Rather the values of these constants may be determined to lie between certain limits.

It appears to be well understood by workers in classical elasticity that a small error in measurement of the elastic constants will not have much effect on the solution to the problem in question, and in problems where the solution can be determined explicitly for arbitrary elastic constants the effect of a small error can be easily determined. However, if the solution cannot be computed but instead pointwise bounds for the solution and its derivatives are sought, one must also take into account the error which might arise due to inaccuracies in the determination of the elastic constants.

In this paper we develop a priori inequalities appropriate to the first and second boundary value problems. These inequalities give upper and lower bounds for the pointwise error in approximation of the exact solution by an arbitrary function. The error in measurement of the elastic constants as well as the error in approximation of the data of the problem is taken into account in the bounds. Thus by choosing trial functions to closely approximate the data, a close approximation can be obtained, with a known bound on the error, provided the error in the constants is also known. The part of the error which results from the fact that the trial function does not satisfy exactly the data of the problem is quadratic. Thus, the Rayleigh-Ritz technique may be employed. This means that we choose from a class of trial functions the best function in the sense that the error term involving the data is minimized (see e.g., [3] ${ }^{1}$ ).

The above mentioned inequalities also are interesting in that they clearly display the fact that the solution to the first (or second) boundary value problem for an incompressible medium (Poisson's ratio, $\sigma$, having the value $1 / 2$ ) as formulated in the literature, is actually the limit of the solution for values of $\sigma<1 / 2$. We show in fact that at points in a given region the solution for $\sigma<1 / 2$ converges with all its derivatives to the classical solution. To the authors' knowledge this fact has not been pointed out in the literature.

*Institute for Fluid Dynamics and Applied Mathematics, University of Maryland, College Park, Md.; consultants at the National Bureau of Standards, Washington, D.C.

' Figures in brackets indicate the literature references at the end of this paper. 


\section{Notation}

Let $D$ be a bounded domain with boundary $\Gamma$ in three dimensions. The displacement vector $u^{\alpha}\left(u_{1}^{\alpha}, u_{2}^{\alpha}, u_{3}^{\alpha}\right)$ corresponding to elastic constants $\mu_{\alpha}$ and $\sigma_{\alpha}$ is assumed to satisfy in $D$ the system of equations

$$
\begin{aligned}
& \Delta u_{i}^{\alpha}=p_{,{ }_{i}}-\mu_{\alpha}^{-1} F_{i}, \quad i=1,2,3 \\
& u_{j, j}^{\alpha}=-c_{\alpha} p^{\alpha} .
\end{aligned}
$$

Here $\Delta$ denotes the Laplace operator, the symbol, $i$, denotes partial differentiation with respect to $x_{i}$ and the summation convention is employed for Latin but not Greek indices. The constant $c_{\alpha}$ is given by

$$
c_{\alpha}=1-2 \sigma_{\alpha},
$$

and $F_{i}$ denotes the body force per unit volume. In terms of $u_{i}^{\alpha}$ and the elastic constants the stress components $\tau_{i j}^{\alpha}$ are expressed as

$$
\tau_{i j}^{\alpha}=\mu_{\alpha}\left[u_{i, j}^{\alpha}+u_{j, i}^{\alpha}-\left(1-c_{\alpha}\right) p^{\alpha} \delta_{i j}\right] .
$$

The physically interesting values of $c_{\alpha}$ lie in the interval

$$
0 \leqslant c_{\alpha}<3 .
$$

In this paper we shall, for simplicity, restrict our attention to the first and the second boundary value problems in classical elasticity. In particular, we assume that in $D$

$$
\begin{array}{ll}
\Delta u_{i}^{\alpha}-p^{\alpha}{ }_{i}=-\mu_{\alpha}^{-1} F_{i} ; & \Delta u_{i}^{\beta}-p^{\beta},{ }_{i}=-\mu_{\beta}^{-1} F_{i} \\
u_{j, j}^{\alpha}=-c_{\alpha} p^{\alpha} \quad ; & u_{j, j}^{\beta}=-c_{\beta} p^{\beta} .
\end{array}
$$

We wish to compare the solutions $u_{i}^{\alpha}$ and $u_{i}^{\beta}$ if one of the following sets of boundary conditions is satisfied:

$$
\begin{gathered}
u_{i}^{\alpha}=u_{i}^{\beta}=f_{i} \text { on } \Gamma \\
\mu_{\alpha}^{-1} \tau_{i j}^{\alpha} n_{j}=\mu_{\beta}^{-1} \tau_{i j}^{\beta} n_{j}=g_{i} \text { on } \Gamma \\
\tau_{i j}^{\alpha} n_{j}=\tau_{i j}^{\beta} n_{j}=h_{i} \text { on } \Gamma .
\end{gathered}
$$

From the point of view of actual physical applicability, problem $(2.5 \mathrm{~A})$ and $(2.5 \mathrm{C})$ are the two interesting cases.

In the subsequent sections we shall set

$$
w_{i}=u_{i}^{\alpha}-u_{i}^{\beta} .
$$

\section{Pointwise Convergence}

We demonstrate in this section that if $p^{\beta}$ and $F_{i}$ are square integrable in $D$ and $h_{i}$ is square integrable on $\Gamma$, then at interior points of $D, u_{i}^{\alpha}$ and all its derivatives tend to $u_{i}^{\beta}$ and its corresponding derivatives as $c_{\alpha} \rightarrow c_{\beta}$, and $\mu_{\alpha} \rightarrow \mu_{\beta}$. 
We assume that there exists a constant $M_{\beta}$ such that

$$
\int_{D}\left[p^{\beta}\right]^{2} d v<M_{\beta}
$$

Let us assume now that we were able to obtain for either of problems (2.5A) to (2.5C), an $L_{2}$ bound for $w_{i}$ in terms of $L_{2}$ integrals of the data of $u_{i}^{\beta}$. Suppose further that the coefficients of the integrals of data tend to zero as $c_{\alpha} \rightarrow c_{\beta}$ and $\mu_{\alpha} \rightarrow \mu_{\beta}$. Then if the data of $u_{i}^{\beta}$ are in $L_{2}$ it follows that as $\alpha \rightarrow \beta, w_{i} \rightarrow 0$ in $L_{2}$. Pointwise convergence would then follow from the continuity of $w_{i}$. The required $L_{2}$ bounds for $w_{i}$ are derived in appendix 1 and appendix 2 . Since we are also interested in computing pointwise bounds, explicit constants will be obtained. In problem (2.5A) we have

$$
\int_{D} w_{i} w_{i} d v \leqslant \frac{M_{\beta}}{2 \lambda_{1} c_{\alpha}}\left(c_{\alpha}-c_{\beta}\right)^{2}+\frac{\left(\mu_{\alpha}^{-1}-\mu_{\beta}^{-1}\right)^{2}}{\lambda_{1}^{2}} \int_{D} F_{i} F_{i} d v
$$

and in problem $(2.5 \mathrm{~B})$

$$
\int_{D} w_{i} w_{i} d v \leqslant \frac{k_{4}}{2} F\left(c_{\alpha}\right) M_{\beta}\left(c_{\alpha}-c_{\beta}\right)^{2}+\left(\mu_{\alpha}^{-1}-\mu_{\beta}^{-1}\right)^{2} k_{4}^{2} \int_{D} F_{i} F_{i} d v
$$

These inequalities follow from (3.1) using (5.23) and (5.30) of appendix 1. For case (2.5C) we have instead from (3.1) and appendix 2,

$$
\begin{aligned}
\int_{D} w_{i} w_{i} d v \leqslant \frac{k_{4}\left(c_{\alpha}-c_{\beta}\right)^{2}}{(2-3 \gamma)\left[1-(\gamma-1) c_{\alpha}\right]} M_{\beta}+k_{4}\left[\frac{k_{1}}{k_{3}} \frac{\left(\mu_{\alpha}^{-1}-\mu_{\bar{\beta}}^{-1}\right)^{2}}{(2-3 \gamma)}\right] \int_{\Gamma} h_{i} h_{i} d s & +\frac{8\left(\mu_{\alpha}^{-1}-\mu_{\beta}^{-1}\right)}{(2-3 \gamma)^{2}} k_{4}^{2} \int_{D} F_{i} F_{i} d v,
\end{aligned}
$$

where $\gamma$ satisfies (5.8).

We wish to obtain from these expressions pointwise inequalities in terms of the appropriate sets of data. In order to do this we introduce the function $\Gamma_{i k}^{\alpha}(P, Q)$, the Kelvin fundamental solution of (2.1); i.e.,

$$
\Gamma_{i k}^{\alpha}(P, Q)=\frac{1}{8 \pi\left(1+c_{\alpha}\right)}\left\{\frac{\left(1+2 c_{\alpha}\right) \delta_{i k}}{r_{P Q}}+\frac{\left[x_{i}(P)-x_{i}(Q)\right]\left[x_{k}(P)-x_{k}(Q)\right]}{r_{P Q}^{3}}\right\}
$$

where $r_{P Q}$ is the distance from the point $P$ to the point $Q$. Then a particular solution $\bar{u}_{i}^{\alpha}$ of (2.1) is given by

$$
\bar{u}_{i}^{\alpha}(P)=\mu_{\alpha}^{-1} \int_{D} \Gamma_{i k}^{\alpha}(P, Q) F_{k} d v_{Q}
$$

Let $u_{i}^{\alpha}$ now be defined as

$$
\hat{u}_{i}^{\alpha}=u_{i}^{\alpha}-\bar{u}_{i}^{\alpha}
$$

Clearly then $\hat{u}_{i}^{\alpha}$ satisfies the system

$$
\Delta \hat{u}_{i}^{\alpha}+\frac{1}{c_{\alpha}} \hat{u}_{j, j i}^{\alpha}=0 \text { in } D .
$$

We now define

$$
w_{i}=\bar{w}_{i}+\hat{w}_{i}
$$


where

$$
\bar{w}_{i}=\bar{u}_{i}^{\alpha}-\bar{u}_{i}^{\beta}=\int_{D}\left[\mu_{\alpha}^{-1} \Gamma_{i k}^{\alpha}(P, Q)-\mu_{\beta}^{-1} \Gamma_{i k}^{\beta}(P, Q)\right] F_{k} d v
$$

and

$$
\hat{w}_{i}=\hat{u}_{i}^{\alpha}-\hat{a}_{i}^{\beta} .
$$

Since $\hat{u}_{i}^{\alpha}$ and $\hat{u}_{i}^{\beta}$ are biharmonic functions, it follows that $\hat{W}_{i}$ is a biharmonic function. We may, therefore, make use of some mean value inequalities for biharmonic functions (see, e.g., Bramble and Payne [2]). That is, if $R$ denotes the radius of the largest sphere contained in $D$ with center at the point at which bounds are desired (we take this point to be the origin), then

$$
\hat{w}_{i}(0) \hat{w}_{i}(0) \leqslant \frac{75}{16 \pi R^{2}} \int_{D} \hat{w}_{i} \hat{w}_{i} d v
$$

Similarly, as is shown in [3], it is possible to obtain

$$
D^{\nu}\left(\hat{w}_{i}\right)(0) D^{\nu}\left(\hat{w}_{i}\right)(0) \leqslant C^{\nu} \int_{D} \hat{w}_{i} \hat{w}_{i} d v
$$

where $D^{\nu}$ is any $\nu$ th order partial derivative and $C^{\nu}$ is a constant depending on $R$ and $\nu$.

It is clear at this point that if in any of problems (2.5A to $2.5 \mathrm{C}$ ) the body force $F_{i}$ is identically zero, then $\bar{w}_{i} \equiv 0$ and $\hat{w}_{i} \equiv w_{i}$. By using the appropriate bound for $\int_{D} w_{i} w_{i} d v$ (given by (3.2), (3.3), or (3.4) we would obtain the desired pointwise bounds for $u_{i}^{\alpha}-u_{i}^{\beta}$. In fact, in any compact subdomain of $D$, we could obtain a uniform bound of the following type for any $\nu$ th order partial derivative, $D^{\nu}\left(u_{i}^{\alpha}-u_{i}^{\beta}\right)$, of $u_{i}^{\alpha}-u_{i}^{\beta}$ (assuming that in (3.5), $\int_{\Gamma} h_{i} h_{i} d s$ is bounded):

$$
\left.D^{\nu}\left(u_{i}^{\alpha}-u_{i}^{\beta}\right) D^{\nu}\left(u_{i}^{\alpha}-u_{i}^{\beta}\right)\right|_{(0,0,0)} \leqslant k_{\nu}\left[c_{\alpha}-c_{\beta}\right]^{2}+K_{\nu}\left[\mu_{\alpha}^{-1}-\mu_{\beta}^{-1}\right]^{2}
$$

In either of problems $(2.5 \mathrm{~A})$ or $(2.5 \mathrm{~B})$, the constant $K_{\nu}$ would in fact be zero. We turn now to the case $F_{i} \not \equiv 0$.

Clearly

$$
w_{i}(0) w_{i}(0) \leqslant 2\left[\bar{w}_{i}(0) \bar{w}_{i}(0)+\hat{w}_{i}(0) \hat{w}_{i}(0)\right]
$$

But from (3.12)

$$
\hat{w}_{i(0)} \hat{w}_{i(0)} \leqslant \frac{75}{8 \pi R^{3}}\left[\int_{D} w_{i} w_{i} d v+\int_{D} \bar{w}_{i} \bar{w}_{i} d v\right]
$$

Combining (3.15) and (3.16) we obtain

$$
w_{i}(0) w_{i}(0) \leqslant \frac{75}{4 \pi R^{3}} \int_{D} w_{i} w_{i} d v+2 \bar{w}_{i}(0) \bar{w}_{i}(0)+\frac{75}{4 \pi R^{3}} \int_{D} \bar{w}_{i} \bar{w}_{i} d v
$$

We seek now an appropriate bound for the last two terms of (3.17). An application of Schwarz's inequality to (3.10) gives

$$
\bar{w}_{i} \bar{w}_{i} \leqslant \int_{D}\left[\mu_{\alpha}^{-1} \Gamma_{i k}^{\alpha}-\mu_{\beta}^{-1} \Gamma_{i k}^{\beta}\right]\left[\mu_{\alpha}^{-1} \Gamma_{i k}^{\alpha}-\mu_{\beta}^{-1} \Gamma_{i k}^{\beta}\right] d v \int_{D} F_{k} F_{k} d v .
$$


We now insert (3.5) into the first term on the right and make use of the arithmetic-geometric mean inequality to obtain

$$
\int_{D}\left[\mu_{\alpha}^{-1} \Gamma_{i k}^{\alpha}-\mu_{\beta}^{-1} \Gamma_{i k}^{\beta}\right]\left[\mu_{\alpha}^{-1} \Gamma_{i k}^{\alpha}-\mu^{-1} \Gamma_{i k}^{\beta}\right] d v \leqslant \frac{G(P)}{32 \pi^{2}}\left\{2\left[\mu_{\alpha}^{-1}-\mu_{\beta}^{-1}\right]^{2}+\left[\frac{\left(1+2 c_{\alpha}\right)}{\mu_{\alpha}\left(1+c_{\alpha}\right)}-\frac{\left(1+2 c_{\beta}\right)}{\mu_{\beta}\left(1+c_{\beta}\right)}\right]^{2}\right\}
$$

where $G(P)=\int_{D} r_{P Q}^{-2} d v_{Q}$.

Combining (3.18) and (3.19) and integrating we have

$$
\int_{D} \bar{w}_{i} \bar{w}_{i} d v \leqslant \frac{H}{32 \pi^{2}}\left\{2\left[\mu_{\alpha}^{-1}-\mu_{\beta}^{-1}\right]^{2}+\left[\frac{\left(1+2 c_{\alpha}\right)}{\mu_{\alpha}\left(1+c_{\alpha}\right)}-\frac{\left(1+2 c_{\beta}\right)}{\mu_{\beta}\left(1+c_{\beta}\right)}\right]^{2}\right\} \int_{D} F_{k} F_{k} d v
$$

where $H=\int_{D} \int_{D} r_{P Q}^{-2} d v_{Q} d v_{P}$

It follows now from (3.18), (3.19), and (3.20) that

$$
\begin{aligned}
& 2 \bar{w}_{i}(O) \bar{w}_{i}(O)+\frac{75}{4 \pi R^{3}} \int_{D} \bar{w}_{i} \bar{w}_{i} d v \leqslant \frac{1}{16 \pi^{2}}\left\{G(O)+\frac{75}{8 \pi R^{3}} H\right\} \\
& \cdot\left\{2\left[\mu_{\alpha}^{-1}-\mu_{\beta}^{-1}\right]^{2}+\left[\frac{\left(1+2 c_{\alpha}\right)}{\mu_{\alpha}\left(1+c_{\alpha}\right)}-\frac{\left(1+2 c_{\beta}\right)}{\mu_{\beta}\left(1+c_{\beta}\right)}\right]^{2}\right\} \int_{D} F_{k} F_{k} d v .
\end{aligned}
$$

The following bounds on $G(0)$ and $H$ are easy to obtain:

$$
G(0) \leqslant(4 \pi d)
$$

and

$$
H \leqslant V(4 \pi d)
$$

where $V$ is the volume of $D$ and $d$ is the diameter. Thus combining (3.17), and (3.21), (3.22), (3.23), and (3.26) we obtain

$$
\begin{aligned}
w_{i}(0) w_{i}(0) \leqslant \frac{75}{4 \pi R^{3}} \int_{D} w_{i} w_{i} d v+\left[\frac{d}{4 \pi}\left\{1+\frac{75}{8 \pi R^{3}} V\right\}\right] \\
\cdot\left\{2\left(\mu_{\alpha}^{-1}-\mu_{\beta}^{-1}\right]^{2}+\left[\frac{\left(1+2 c_{\alpha}\right)}{\mu_{\alpha}\left(1+c_{\alpha}\right)}-\frac{\left(1+2 c_{\beta}\right)}{\mu_{\alpha}\left(1+c_{\alpha}\right)}\right]^{2}\right\} \int_{D} F_{k} F_{k} d v
\end{aligned}
$$

If $F_{k}$ is square integrable then the insertion of (3.2), (3.3), or (3.5) into (3.24) gives in any compact subdomain $D_{1}$ of $D$ a uniform bound of the following type.

$$
\left[u_{i}^{\alpha}(P)-u_{i}^{\beta}(P)\right]\left[u_{i}^{\alpha}(P)-u_{i}^{\beta}(P)\right] \leqslant B_{1}\left[\mu_{\alpha}^{-1}-\mu_{\beta}^{-1}\right]^{2}+B_{2}\left[c_{\alpha}-c_{\beta}\right]^{2}
$$

where $B_{1}$ and $B_{2}$ are independent of the point $P$ in $D_{1}$ and can be chosen independent of the elastic constants. This follows because of the fact that since $c_{\alpha}, c_{\beta} \geqslant 0$,

$$
\begin{aligned}
{\left[\frac{\left(1+2 c_{a}\right)}{\mu_{\alpha}\left(1+c_{\alpha}\right)}-\frac{\left(1+2 c_{\beta}\right)}{\mu_{\beta}\left(1+c_{\beta}\right)}\right]^{2}=\left\{\left(\mu_{\alpha}^{-1}-\mu_{\beta}^{-1}\right) \frac{1+2 c_{\alpha}}{1+c_{\alpha}}-\mu_{\beta}^{-1} \frac{\left(c_{\alpha}-c_{\beta}\right)}{\left(1+c_{\alpha}\right)\left(1+c_{\beta}\right)}\right\}^{2} } & \\
& \leqslant 2\left\{4\left(\mu_{\alpha}^{-1}-\mu_{\beta}^{-1}\right)^{2}+\mu_{\beta}^{-1}\left(c_{\alpha}-c_{\beta}\right)^{2}\right\}
\end{aligned}
$$

The inequality (3.25) clearly implies the uniform convergence of $u_{i}^{\alpha}$ to $u_{i}^{\beta}$ in any compact subdomain of $D$ as $\mu_{\alpha} \rightarrow \mu_{\beta}$ and $c_{\alpha} \rightarrow c_{\beta}$. 
It is clear that if $F_{k}$ has a sufficient number of square integrable derivatives then the convergence of the derivatives of $u_{i}^{\alpha}$ may be obtained in a similar way. To see this we note that

$$
\frac{\partial \Gamma_{i k}^{\alpha}(P, Q)}{\partial x_{P}} \equiv-\frac{\partial \Gamma_{i k}^{\alpha}(P, Q)}{\partial x_{Q}} .
$$

Thus (3.6) may be differentiated with respect to the variables of the point $P$, the order of differentiation and integration being interchanged on the right. Then (3.27) is used followed by an integration by parts which takes the differentiation off of $\Gamma_{i k}^{\alpha}$. The bounds for $\left|u_{i, \rho}^{\alpha}(P)\right|$ are then obtained in a manner similar to that used in obtaining (3.25). To obtain the desired expressions for higher derivatives, we successively differentiate $\Gamma_{i k}^{\alpha}$ and use (3.27) to throw the differentiation off of $\Gamma_{i k}^{\alpha}$ in the volume integral on the right. Then if a sufficient number of derivatives of $F_{k}$ are square integrable over $D$ and over $\Gamma$ we obtain a uniform bound similar to (3.25) for any desired derivative of $u_{i}^{\alpha}$. This shows, then, that $u_{i}^{\alpha}$ and its derivatives converge to $u_{i}^{\beta}$ and its derivatives.

We note, in particular, that if $c_{\beta}=0$ the $u_{i}^{\beta}$ problem is the classical boundary value problem for an incompressible medium. It follows then that as $c_{\alpha} \rightarrow 0$ and $\mu_{\alpha} \rightarrow \mu_{\beta}$ the solution to the problem corresponding to $\alpha$ converges to the solution in the incompressible case. This fact, to the knowledge of the authors, has never been pointed out in the literature.

Another interesting fact is that $F_{k}$ need only have the desired number of square integrable derivatives in a neighborhood of the point at which convergence is sought. The procedures used in this section could be applied to any neighborhood $\Omega$ of the origin. Then since

$$
\int_{\Omega} w_{i} w_{i} d v \leqslant \int_{D} w_{i} w_{i} d v
$$

it would follow that if $F_{k}$ were square integrable over $D$ and enough derivatives of $F_{k}$ were square integrable over $\Omega$ and its boundary, uniform convergence in $\Omega$ of the derivative would follow.

\section{Effect of Error in Measurement of Elastic Constants on Pointwise Bounds}

We consider now problem (2.1) with either the displacement components or the surface tractions prescribed on $\Gamma$. Since $c_{\alpha}$ and $\mu_{\alpha}$ must be determined empirically we shall assume that some error was made in making the measurements and that the values $c_{\beta}$ and $\mu_{\beta}$ were actually obtained. If $\varphi_{i}$ is a sufficiently smooth vector function chosen to approximate the solution $u_{i}^{\alpha}$ we may write

$$
\left|u_{i}^{\alpha}-\varphi_{i}\right| \leqslant\left|u_{i}^{\alpha}-u_{i}^{\beta}\right|+\left|u_{i}^{\beta}-\varphi_{i}\right| .
$$

We wish to obtain upper and lower bounds for $u_{i}^{\alpha}(0)$. If we can make each term on the right of (4.1) less than some prescribed $\epsilon$ we would then have

$$
-2 \epsilon<u_{i}^{\alpha}-\varphi_{i}<2 \epsilon
$$

and $\varphi_{i}$ would be a good pointwise approximation to $u_{i}^{\alpha}$.

The bound for the first term on the right of (4.1) is obtained for the first boundary value problem in elasticity from (3.24) and (5.22) of appendix 1.

$$
\left(u_{i}^{\alpha}(0)-u_{i}^{\beta}(0)\right)\left(u_{i}^{\alpha}(0)-u_{i}^{\beta}(0)\right) \leqslant \frac{75}{4 \pi R^{3}}\left\{A_{\alpha \beta} \int_{D} F_{i} F_{i} d v+\frac{3\left(c_{\alpha}-c_{\beta}\right)^{2} \mu_{\beta}^{-1} E\left(u^{\beta}, u^{\beta}\right)}{\lambda_{1} c_{\alpha} c_{\beta}\left(3-c_{\beta}\right)}\right\},
$$

where

$$
A_{\alpha \beta}=\left(1 / \lambda_{1}^{2}+2 R^{3} d / 15+5 V d / 4 \pi\right)\left(\mu_{\alpha}^{-1}-\mu_{\beta}^{-1}\right)^{2}+d \mu_{\beta}^{-1}\left(2 R^{3} / 75+V / 4 \pi\right)\left(c_{\alpha}-c_{\beta}\right)^{2} .
$$


A bound for $E\left(u^{\beta}, u^{\beta}\right)$ is obtained by the methods of Diaz and Greenberg [4], Fichera [6], Synge [10], or Bramble and Payne [3, eq (3.12)].

The second term on the right of (4.1) may be dealt with by the method of Bramble and Payne [3, eq (4.10)].

We look now at the stress components $\tau_{i j}^{\alpha}$. Using the triangle inequality we obtain

$$
\left|\tau_{i j}^{\alpha}-\tau_{i j}\right| \leqslant\left|\tau_{i j}^{\alpha}-\tau_{i j}^{\beta}\right|+\left|\tau_{i j}^{\beta}-\tau_{i j}\right|
$$

where

$$
\tau_{i j}=\mu_{\beta}\left[\varphi_{i, j}+\varphi_{j, i}+\frac{\left(1-c_{\beta}\right)}{c_{\beta}} \varphi_{j, j} \delta_{i j}\right]
$$

is an approximation to $\tau_{i j}^{\alpha}$. Bounds for the first term on the right of (4.4) may be obtained using (3.13) following again the method outlined at the end of section 3 . The second term on the right may be dealt with as described in [3].

For the second boundary value problem in elasticity we again use (4.1) and (4.4), where in this case $u_{i}^{\alpha}$ and $u_{i}^{\beta}$ are solutions to (2.5C) and bounds for the second term on the right are given in [1].

\section{Appendix 1}

In appendix 1 we derive the inequalities (5.22), (5.23), (5.29), and (5.30) which are needed in the text. In order to do this we start with

$$
\int_{D} w_{i, j}\left(w_{i, j}+w_{j, i}\right) d v
$$

In particular, we bound this expression in terms of the solution of the problem with elastic constants $\mu_{\beta}, c_{\beta}$. Consider then the Green's identity

$$
\int_{D} w_{i, j}\left(w_{i, j}+w_{j, i}\right) d v=\int_{\Gamma} w_{i}\left(w_{i, j}+w_{j, i}\right) n_{j} d s-\int_{D} w_{i}\left(\Delta w_{i}+w_{j, j i}\right) d s .
$$

Making use of (2.5) and integrating by parts we obtain for $w_{i}$ satisfying (2.6)

$$
\begin{aligned}
& \int_{D} w_{i, j}\left(w_{i, j}+w_{j, i}\right) d v=-\int_{\Gamma} w_{i}\left\{\mu_{\alpha}^{-1} \tau_{i j}^{\alpha} n_{j}-\mu_{\beta}^{-1} \tau_{i j}^{\beta} n_{j}\right\} d s+\left(u_{\alpha}^{-1}-\mu_{\beta}^{-1}\right) \int_{D} w_{i} F_{i} d v \\
&+\left(c_{\alpha}+c_{\beta}-2 c_{\alpha} c_{\beta}\right) \int_{D} p^{\alpha} p^{\beta} d v-c_{\alpha}\left(1-c_{\alpha}\right) \int_{D}\left(p^{\alpha}\right)^{2} d v-c_{\beta}\left(1-c_{\beta}\right) \int_{D}\left(p^{\beta}\right)^{2} d v .
\end{aligned}
$$

In either of problems $(2.5 \mathrm{~A})$ or $(2.5 \mathrm{~B})$, the boundary integral on the right of (5.2) will vanish. In fact, this integral would vanish for various mixed boundary conditions which are encountered in physical situations. On the other hand, in problem (2.5C) the boundary term does not vanish. We shall thus consider first problems $(2.5 \mathrm{~A})$ and $(2.5 \mathrm{~B})$ and then treat $(2.5 \mathrm{C})$ at the end of the section. If $0<c_{\alpha} \leqslant 3 / 4(\sigma \geqslant 1 / 8)$ we rewrite (5.2) for (A) and (B) as

$$
\begin{aligned}
\int_{D} w_{i, j}\left(w_{i, j}+w_{j, i}\right) d v=-c_{\alpha}\left(1-c_{\alpha}\right) \int_{D}\left[p^{\alpha}\right. & \left.-\frac{\left(c_{\alpha}+c_{\beta}-2 c_{\alpha} c_{\beta}\right)}{2 c_{\alpha}\left(1-c_{\alpha}\right)} p^{\beta}\right]^{2} d v \\
& +\left(\mu_{\alpha}^{-1}-\mu_{\alpha}^{-1}\right) \int_{D} w_{i} F_{i} d v+\frac{\left(c_{\alpha}-c_{\beta}\right)^{2}}{4 c_{\alpha}\left(1-c_{\alpha}\right)} \int_{D}\left(p^{\beta}\right)^{2} d v
\end{aligned}
$$

The first term on the right is nonpositive. Thus

$$
\int_{D} w_{i, j}\left(w_{i, j}+w_{j, i}\right) d v \leqslant \frac{\left(c_{\alpha}-c_{\beta}\right)^{2}}{4 c_{\alpha}\left(1-c_{\alpha}\right)} \int_{D}\left(p^{\beta}\right)^{2} d v+\left(\mu_{\alpha}^{-1}-\mu_{\beta}^{-1}\right) \int_{D} w_{i} F_{i} d v
$$


The strain energy $E\left(u^{\beta}, u^{\beta}\right)$ corresponding to $u^{\beta}$ is given by:

$$
2 \mu_{\beta}^{-1} E\left(u^{\beta}, u^{\beta}\right)=\int_{D}\left[u_{i, j}^{\beta}\left(u_{i, j}^{\beta}+u_{j, i}^{\beta}\right)+c_{\beta}\left(1-c_{\beta}\right)\left(p^{\beta}\right)^{2}\right] d v . \geqslant \frac{c_{\beta}}{3}\left(3-c_{\beta}\right) \int_{D}\left(p^{\beta}\right)^{2} d v .
$$

The last inequality results from the fact that

$$
u_{i, j}^{\beta}\left(u_{1, j}^{\beta}+u_{j, i}^{\beta}\right) \leqslant 2\left[\left(u_{1,1}^{\beta}\right)^{2}+\left(u_{2,2}^{\beta}\right)^{2}+\left(u_{3,3}^{\beta}\right)^{2}\right] \geqslant 2 / 3\left[u_{i, i}^{\beta}\right]^{2} .
$$

Hence if $c_{\beta} \neq 0$ we have from (5.4) and (5.5)

$$
\int_{D} w_{i, j}\left(w_{i, j}+w_{j, i}\right) d v \geqslant \frac{3 \mu_{\beta}^{-1}\left(c_{\alpha}-c_{\beta}\right)^{2}}{2 c_{\alpha} c_{\beta}\left(1-c_{\alpha}\right)\left(3-c_{\beta}\right)} E\left(u^{\beta}, u^{\beta}\right)+\left(\mu_{\alpha}^{-1}-\mu_{\beta}^{-1}\right) \int_{D} w_{i} F_{i} d v .
$$

Bounds for $E\left(u^{\beta}, u^{\beta}\right)$ in problems (2.5A) and (2.5B) in terms of the data of the problem have been given for instance by Diaz and Greenberg [4], Fichera [6], Prager and Synge [9], Synge [10], and Bramble and Payne [1,3]. If $F_{i} \equiv 0$ such bounds would give then for $0<c_{\alpha} \leqslant 3 / 4$ an upper bound for

$$
\int_{D} w_{i, j}\left(w_{i, j}+w_{j, i}\right) d v
$$

in terms of the data of the $u_{i}^{\beta}$ problem of $(2.5 \mathrm{~A})$ or $(2.5 \mathrm{~B})$.

For $c_{\alpha}>3 / 4$ we choose a constant $\gamma$ in the interval

$$
1-c_{\alpha}^{-1}<\gamma<2 / 3
$$

and consider the following identity derived from (5.2) by addition of the appropriate term to each side of the equation

$$
\begin{aligned}
& \int_{D} w_{i, j}\left(w_{i, j}+w_{j, i}\right) d v-\gamma \int_{D} w_{j, j}^{2} d v=\left[c_{\alpha}+c_{\beta}+2(\gamma-1) c_{\alpha} c_{\beta}\right] \int_{D} p^{\alpha} p^{\beta} d v \\
& \quad-c_{\alpha}\left\{1+(\gamma-1) c_{\alpha}\right\} \int_{D}\left(p^{\alpha}\right)^{2} d v-c_{\beta}\left\{1-(\gamma-1) c_{\beta}\right\} \int_{D}\left(p^{\beta}\right)^{2} d v+\left(\mu_{\alpha}^{-1}-\mu_{\beta}^{-1}\right) \int_{D} w_{i} F_{i} d v .
\end{aligned}
$$

Using (5.6) then we have

$$
\begin{aligned}
\left(1-\frac{3 \gamma}{2}\right) \int_{D} w_{i, j}\left(w_{i, j}+w_{j, i}\right) d v \leqslant & -c_{\alpha}\left\{1+(\gamma-1) c_{\alpha}\right\} \int_{D}\left\{p^{\alpha}+\frac{\left[c_{\alpha}+c_{\beta}+2(\gamma-1) c_{\alpha} c_{\beta}\right]}{2 c_{\alpha}\left[1+(\gamma-1) c_{\alpha}\right]} p^{\beta}\right\}^{2} d v \\
& +\frac{\left(c_{\alpha}-c_{\beta}\right)^{2}}{4 c_{\alpha}\left[1+(\gamma-1) c_{\alpha}\right]} \int_{D}\left(p^{\beta}\right)^{2} d v+\left(\mu_{\alpha}^{-1}-\mu_{\beta}^{-1}\right) \int_{D} w_{i} F_{i} d v .
\end{aligned}
$$

Thus we obtain

$$
\int_{D} w_{i, j}\left(w_{i, j}+w_{j, i}\right) d v \leqslant \frac{\left(c_{\alpha}-c_{\beta}\right)^{2}}{2(2-3 \gamma) c_{\alpha}\left[1+(\gamma-1) c_{\alpha}\right.} \int_{D}\left[p^{\beta}\right]^{2} d v+\frac{2\left(\mu_{\alpha}^{-1}-\frac{\left.\mu^{-1}\right)}{(2-3} \frac{\beta}{\gamma}\right)}{2} \int_{D} w_{i} F_{i} d v .
$$

The choice $\gamma=2 c_{\alpha} /\left(3+2 c_{\alpha}\right)$ yields

$$
\int_{D} w_{i, j}\left(w_{i, j}+w_{j, i}\right) d v \leqslant \frac{\left(3+2 c_{\alpha}\right)^{2}\left(c_{\alpha}-c_{\beta}\right)^{2}}{4 c_{\alpha}\left(3-c_{\alpha}\right)^{2}} \int_{D}\left(p^{\beta}\right)^{2} d v+\left(\mu_{\alpha}^{-1}-\mu_{\beta}^{-1}\right) \int_{D} w_{i} F_{i} d v .
$$

Proceeding as before we obtain finally the inequality

$$
\int_{D} w_{i, j}\left(w_{i, j}+w_{i, i}\right) d v \leqslant F\left(c_{\alpha}\right)\left(c_{\alpha}-c_{\beta}\right)^{2} \int_{D}\left(p^{\beta}\right)^{2} d v+\left(\mu_{\alpha}^{-1}-\mu_{\beta}^{-1}\right) \int_{D} F_{i} w_{i} d v
$$


where

$$
F\left(c_{\alpha}\right)=\left\{\begin{array}{l}
{\left[4 c_{\alpha}\left(1-c_{\alpha}\right)\right]^{-1}, 0<c_{\alpha} \leqslant 3 / 4} \\
{\left[\left(3+2 c_{\alpha}\right)^{2}\left[4 c_{\alpha}\left(3-c_{\alpha}\right)^{2}\right]^{-1}, c_{\alpha}>3 / 4\right.}
\end{array}\right.
$$

It follows then that for $c_{\beta} \neq 0$

$$
\int_{D} w_{i, j}\left(w_{i, j}+w_{j, i}\right) d v \leqslant \frac{6 \mu_{\beta}^{-1} F\left(c_{\alpha}\right)}{c_{\beta}\left(3-c_{\beta}\right)}\left(c_{\alpha}-c_{\beta}\right)^{2} E\left(u^{\beta}, u^{\beta}\right)+\left(\mu_{\alpha}^{-1}-\mu_{\beta}^{-1}\right) \int_{D} w_{i} F_{i} d v .
$$

In problem $(2.5 \mathrm{~A})$ we have the relation

$$
\begin{aligned}
\int_{D} w_{i, j} w_{i, j} d v=-\int_{D} w_{i} \Delta w_{i} d v=-\int_{D} & w_{i}\left(p^{\alpha}-p^{\beta}\right) d v \\
& =-\int_{D}\left(c_{\alpha} p^{\alpha}-c_{\beta} p^{\beta}\right)\left(p^{\alpha}-p^{\beta}\right) d v+\left(\mu_{\alpha}^{-1}-\mu_{\beta}^{-1}\right) \int_{D} F_{i} w_{i} d v .
\end{aligned}
$$

or

$$
\int_{D} w_{i, j} w_{i, j} d v=-c_{\alpha} \int_{D}\left[p^{\alpha}-\frac{\left(c_{\alpha}+c_{\beta}\right)}{2 c_{\beta}} p^{\beta}\right]^{2} d v+\left(\mu_{\alpha}^{-1}-\mu_{\bar{\beta}^{1}}\right) \int_{D} F_{i} w_{i} d v+\frac{\left(c_{\alpha}-c_{\beta}\right)^{2}}{4 c_{\alpha}} \int_{D}\left(p^{\beta}\right)^{2} d v .(5
$$

Thus, dropping the first term on the right,

$$
\int_{D} w_{i, j} w_{i, j} d v \leqslant \frac{\left(c_{\alpha}-c_{\beta}\right)^{2}}{4 c_{\alpha}} \int_{D}\left(p^{\beta}\right)^{2} d v+\left(\mu_{\alpha}^{-1}-\mu_{\beta}^{-1}\right) \int_{D} F_{i} w_{i} d v
$$

and if $c_{\beta} \neq 0$

$$
\int_{D} w_{i, j} w_{i, j} d v \leqslant 3 \mu_{\beta}^{-1} \frac{\left(c_{\alpha}-c_{\beta}\right)^{2}}{2 c_{\alpha} c_{\beta}\left(3-c_{\beta}\right)} E\left(\mu^{\beta}, \mu^{\beta}\right)+\left(\mu_{\alpha}^{+1}-\mu_{\beta}^{-1}\right) \int_{D} F_{i} w_{i} d v
$$

We have retained the term $\left(\mu_{\alpha}^{-1}-\mu_{\beta}^{-1}\right) \int_{D} F_{i} w_{i} d v$ up to this point since in the important case $F_{i} \equiv 0$ this term will vanish and the inequality will not be altered. Let us assume now that $F_{i} \not \equiv 0$ and note that

$$
\int_{D} w_{i} w_{i} d v \leqslant \frac{1}{\lambda_{1}} \int_{D} w_{i, j} w_{i, j} d v \leqslant \frac{3\left(c_{\alpha}-c_{\beta}\right)^{2} \mu_{\beta}^{-1} E\left(u_{\beta}, u_{\beta}\right)}{2 \lambda_{1} c_{\alpha} c_{\beta}\left(3-c_{\beta}\right)}+\frac{\left(\mu_{\alpha}^{-1}-\mu_{\beta}^{-1}\right)}{\lambda_{1}} \int_{D} F_{i} w_{i} d v
$$

Where $\lambda_{1}$ is the first eigenvalue in the three dimensional fixed membrane problem for $D$. A lower bound for $\lambda_{1}$ is given by the Faber-Krahn inequality [5, 7], i.e.,

$$
\lambda_{1} \geqslant \pi^{2}\left[\frac{4 \pi}{3 V}\right]^{2 / 3}
$$

where $V$ denotes the volume of $D$. From the arithmetic-geometric mean inequality applied to the last term of (5.20) it follows that

$$
\int_{D} w_{i} w_{i} d v \leqslant \frac{3\left(c_{\alpha}-c_{\beta}\right)^{2} \mu_{\beta}^{-1}}{\lambda_{1} c_{\alpha} c_{\beta}\left(3-c_{\beta}\right)} E\left(u_{\beta}, u_{\beta}\right)+\frac{\left(\mu_{\alpha}^{-1}-\mu_{\beta}^{-1}\right)^{2}}{\lambda_{1}^{2}} \int_{D} F_{i} F_{i} d v .
$$

Similarly if we insert (5.18) into the first inequality in (5.20) and apply the arithmetic-geometric mean inequality to the last term we obtain

$$
\int_{D} w_{i} w_{i} d v \leqslant \frac{\left(c_{\alpha}-c_{\beta}\right)^{2}}{2 \lambda_{1} c_{\alpha}} \int_{D}\left(p^{\beta}\right)^{2} d v+\frac{\left(\mu_{\alpha}^{-1}-\mu_{\beta}^{-1}\right)^{2}}{\lambda_{1}^{2}} \int_{D} F_{i} F_{i} d v .
$$


For problem (3.5B) it was shown in $\left[1\right.$, eq (3.14)] that if $w_{i}$ is normalized so that $\int_{\Gamma} w_{i} d s=0$ then

$$
\int_{\Gamma} w_{i} w_{i} d s \leqslant \frac{k_{1}}{2 k_{3}} \int_{D} w_{i, j}\left(w_{i, j}+w_{j, i}\right) d v
$$

where $k_{1}$ and $k_{3}$ are constants given in [1, eqs (2.9) and (2.19)], which are computable in terms of the geometry of $D$.

We now make use of the identity

$$
5 \int_{D} w_{i} w_{i} d v=\int_{\Gamma} x^{k} w_{i}\left(2 n_{i} w_{k}+n_{k} w_{i}\right) d s+2 \int_{D} x^{k} w_{i}\left(w_{i, k}+w_{k, i}\right) d v+2 \int_{D} x^{k} w_{k} w_{i, i} d v
$$

An application of the arithmetic geometric mean inequality and (3.8) then yields (see e.g., [1, eqs (2.26) to $(2.28)])$

$$
\int_{D} w_{i} w_{i} d v \leqslant \frac{6 r_{M}}{5} \int_{\Gamma} w_{i} w_{i} d s+\frac{28 r_{M}^{2}}{25} \int_{D} w_{i, j}\left(w_{i, j}+w_{j, i}\right) d v
$$

where $r_{M}$ denotes the maximum distance from the origin to $\Gamma$. From (5.24) and (5.26) it follows then that

$$
\int_{D} w_{i} w_{i} d v \leqslant k_{4} \int_{D} w_{i, j}\left(w_{i, j}+w_{j, i}\right) d v
$$

where

$$
k_{4}=\left[\frac{3}{5} r_{M} k_{1} / k_{3}+\frac{28}{25} r_{M}^{2}\right] .
$$

Thus if in $(2.5 \mathrm{~B}) c_{\beta} \neq 0$,

$$
\int_{D} w_{i} w_{i} d v \leqslant \frac{12 k_{4} \mu_{\beta}^{-1} F\left(c_{\alpha}-c_{\beta}\right)^{2}}{c_{\beta}\left(3-c_{\beta}\right)} E\left(u^{\beta}, u^{\beta}\right)+\left(\mu_{\alpha}^{-1}-\mu_{\beta}^{-1}\right)^{2} k_{4}^{2} \int_{D} F_{i} F_{i} d v .
$$

Finally, after inserting (5.13) into (5.27) and using the arithmetic-geometric mean inequality on the last term we have

$$
\int_{D} w_{i} w_{i} d v \leqslant 2 k_{4} F\left(c_{\alpha}\right)\left(c_{\alpha}-c_{\beta}\right)^{2} \int_{D}\left(p^{\beta}\right)^{2} d v+k_{4}^{2}\left(\mu_{\alpha}^{-1}-\mu_{\beta}^{-1}\right)^{2} \int_{D} F_{i} F_{i} d v .
$$

\section{Appendix 2}

We derive here inequalities (6.3) and (6.4) which are needed to treat problem (2.5C).

Again we start with $\int_{D} w_{i, j}\left(w_{i, j}+w_{j, i}\right) d v$ and instead of (5.2) we obtain

$$
\begin{aligned}
\int_{D} w_{i, j}\left(w_{i, j}+w_{j, i}\right) d v & =-\int_{\Gamma} w_{i} h_{i}\left[\mu_{\alpha}^{-1}-\mu_{\beta}^{-1}\right] d s+\left(c_{\alpha}+c_{\beta}-2 c_{\alpha} c_{\beta}\right) \int_{D} p^{\alpha} p^{\beta} d v \\
& -c_{\alpha}\left(1-c_{\alpha}\right) \int_{D}\left(p^{\alpha}\right)^{2} d v-c_{\beta}\left(1-c_{\beta}\right) \int_{D}\left(p^{\beta}\right)^{2} d v+\left(\mu_{\alpha}^{-1}-\mu_{\beta}^{-1}\right) \int_{D} w_{i} F_{i} d v
\end{aligned}
$$

which follows from a calculation involving the use of the divergence theorem. We thus obtain as before

$$
\begin{aligned}
\int_{D} w_{i, j}\left(w_{i, j}+w_{j, i}\right) d v & +\frac{2}{(2-3 \gamma)}\left[\mu_{\alpha}^{-1}-\mu_{\beta}^{-1}\right] \int_{\Gamma} w_{i} h_{i} d s \\
& -\frac{2}{(2-3 \gamma)}\left(\mu_{\alpha}^{-1}-\mu_{\beta}^{-1}\right) \int_{D} F_{i} w_{i} d v \leqslant \frac{\left(c_{\alpha}-c_{\beta}\right)^{2}}{2(2-3 \gamma)\left[1+(\gamma-1) c_{\alpha}\right]} \int_{D}\left(p^{\beta}\right)^{2} d v
\end{aligned}
$$


where $\gamma$ satisfies (5.8). It follows then from the arithmetic-geometric mean inequality, (5.24), and (5.27) that if $w_{i}$ is normalized so that $\int_{\Gamma} w_{i} d s=0$ then

$$
\begin{aligned}
& \left\lceil l-\frac{a}{2}-\frac{b}{2}\right] \int_{D} w_{i, j}\left(w_{i, j}+w_{j, i}\right) d v \leqslant \frac{\left[\mu_{\alpha}^{-1}-\mu_{\beta}^{-1}\right]^{2}}{4(2-3 \gamma)^{2} a}\left(k_{1} / k_{3}\right)^{2} \int_{\Gamma} h_{i} h_{i} d s \\
& +\frac{\left(c_{\alpha}-c_{\beta}\right)^{2} \int_{D}\left(p^{\beta}\right)^{2} d v}{2(2-3 \gamma)\left[1+(\gamma-1) c_{\alpha}\right]}+\frac{2\left(\mu_{\alpha}^{-1}-\mu_{\beta}^{-1}\right)^{2}}{(2-3 \gamma)^{2} b} k^{4} \int_{D} F_{i} F_{i} d v
\end{aligned}
$$

for any positive numbers $a$ and $b$. In particular for $a=1 / 2, b=1 / 2(6.2)$ becomes

$$
\begin{aligned}
\int_{D} w_{i, j}\left(w_{i, j}+w_{j, i}\right) d v \leqslant & {\left[\frac{k_{1}}{k_{3}} \frac{\left(\mu_{\alpha}^{-1}-\mu_{\beta}^{-1}\right)}{(2-3 \gamma)}\right]^{2} \int_{\Gamma} h_{i} h_{i} d s } \\
& +\frac{8\left(\mu_{\alpha}^{-1}-\mu_{\beta}^{-1}\right)^{2}}{(2-3 \gamma)^{2}} k_{4} \int_{D} F_{i} F_{i} d v+\frac{\left(\mathbf{c}_{\alpha}-c_{\beta}\right)^{2}}{(2-3 \gamma)\left[1+(\gamma-1) c_{\alpha}\right]} \int_{D}\left(p^{\beta}\right)^{2} d v .
\end{aligned}
$$

As before inserting (6.3) into (5.27) we have

$$
\begin{aligned}
\int_{D} w_{i} w_{i} d v \leqslant k_{4}\left[\frac{k_{1}}{k_{3}} \frac{\left(\mu_{\alpha}^{-1}-\mu_{\beta}^{-1}\right)}{(2-3 \gamma)}\right]^{2} \int_{\Gamma} h_{i} h_{i} d s+\frac{8\left(\mu_{\alpha}^{-1}-\mu_{\beta}^{-1}\right)^{2}}{(2-3 \gamma)^{2}} \int_{D} F_{i} F_{i} d v & \\
& +\frac{k_{4}\left(c_{\alpha}-c_{\beta}\right)^{2}}{(2-3 \gamma)\left[1+(\gamma-1) c_{\alpha}\right]} \int_{D}\left(p^{\beta}\right)^{2} d v .
\end{aligned}
$$

\section{References}

[1] Bramble, J. H. and Payne, L. E. Some inequalities for vector functions with applications in elasticity, Arch. Rat. Mech. 11, No. 1, pp. 16-26 (1962).

[2] Bramble, J. H. and Payne, L. E. Pointwise bounds in the first biharmonic boundary value problem (to appear in J. Math. Phys.).

[3] Bramble, J. H. and Payne, L. E. A priori bounds in the first boundary value problem in elasticity, J. Research NBS 65B (Math. and Math. Phys.) No. 4, pp. 269-276 (1961).

[4] Diaz, J. B. and Greenberg, H. J. Upper and lower bounds for the solution of the first boundary value problem of elasticity, Quart. Appl. Math. 6, pp. 326-331 (1948).

[5] Faber, G., Beweis, dass unter allen homogenen Membranen von gleicher Fläche und gleicher Spannung die Kreisförmige den tiefsten Grundton gibt, Sitzber. Bayer Akad. Wiss., pp. 169-172 (1923).

[6] Fichera, G. Sull'esistenza e sul calcolo delle soluzioni dei problem al contorno, relativi all' equilibrio di un corpo elastico, Annali di Pisa 4, (1950); see also Methods of functional analysis in mathematical physics, Proc. Intr. Cong. Math. Amsterdam 3, pp. 273-296 (1954).

[7] Krahn, E. Über eine von Rayleigh formulierte Minimaleigenschaft des Kreises, Math. Ann. 94, pp. 97-100, (1924); see also Über Minimaleigenschaften der Kugel in drei und mehr Dimensionen, Acta. Comm. Univ. Dorp. A9, pp. 1-44 (1926).

[8] Nicolesco, M. Les fonctiones polyharmonique, Hermann, Paris (1936).

[9] Prager, W. and Synge, J. L. Approximations in elasticity based on the concept of function space, Quart. Appl. Math. 5, pp. 241-269 (1947).

[10] Synge, J. L. Upper and lower bounds for the solutions of problems in elasticity, Proc. Roy. Iris Acad. 53, pp. 41-64. (1950).

(Paper 67B3-100) 